\title{
Thalamocortical Bursts Trigger Recurrent Activity in Neocortical Networks: Layer 4 as a Frequency-Dependent Gate
}

\author{
Michael Beierlein, ${ }^{1}$ Christopher P. Fall, ${ }^{1,2}$ John Rinzel,,2 and Rafael Yuste ${ }^{1}$ \\ ${ }^{1}$ Department of Biological Sciences, Columbia University, New York, New York 10027, and ${ }^{2}$ Center for Neural Science, \\ New York University, New York, New York 10003
}

\begin{abstract}
Sensory information reaches the cortex via thalamocortical (TC) synapses in layer 4 . Thalamic relay neurons that mediate information flow to cortex operate in two distinct modes, tonic and burst firing. Burst firing has been implicated in enhancing reliability of information flow between individual neurons. However, little is known about how local networks of neocortical neurons respond to different temporal patterns of TC activity. We studied cortical activity patterns evoked by stimulating TC afferents at different frequencies, using a combination of electrophysiology and calcium imaging in TC slices that allowed for the reconstruction of spatiotemporal activity with single-cell resolution. Stimulation of TC axons at low frequencies triggered action potentials in only a small number of layer 4 neurons. In
\end{abstract}

contrast, brief high-frequency stimulus trains triggered widespread recurrent activity in populations of neurons in layer 4 and then spread into adjacent layers $2 / 3$ and 5. Recurrent activity had a clear threshold, typically lasted $300 \mathrm{msec}$, and could be evoked repetitively at frequencies up to $0.5 \mathrm{~Hz}$. Moreover, the spatial extent of recurrent activity was controlled by the TC pattern of activity. Recurrent activity triggered within the highly interconnected networks of layer 4 might act to selectively amplify and redistribute transient high-frequency TC inputs, filter out low-frequency inputs, and temporarily preserve a record of past sensory activity.

Key words: fura-2; barrel cortex; feedforward inhibition; temporal summation; NMDA; persistent activity
Neocortex transforms sensory inputs into complex spatiotemporal patterns of activity (Mountcastle, 1998). The barrel field of rodent somatosensory cortex provides a unique model system for the study of such transformations (Woolsey and Van der Loos, 1970). Anatomical and physiological studies have demonstrated that the connectivity between excitatory neurons within a barrel is high (Feldmeyer et al., 1999; Petersen and Sakmann, 2000), whereas neurons in different barrels are rarely interconnected. Indeed, in vitro voltage-sensitive dye imaging studies have shown that activity evoked in thalamocortical (TC) fibers (Laaris et al., 2000), white matter (Contreras and Llinas, 2001), or directly in single barrels (Petersen and Sakmann, 2001; Laaris and Keller, 2002) remains primarily confined to a single barrel-related column. The high degree of connectivity within layer 4 networks, together with the distinct properties of synapses interconnecting excitatory neurons (Fleidervish et al., 1998), could serve to amplify sensory-evoked activity (Douglas et al., 1995). At the same time, synaptic inhibition likely plays an important role in shaping thalamocortical (Simons, 1978) and intracortical activity. Thalamic afferents form strong connections onto interneurons (Gibson et al., 1999; Porter et al., 2001; Swadlow and Gusev, 2001), suggesting that feedforward inhibition tightly regulates sensoryevoked activity. Furthermore, intracortical information flow is controlled by numerous types of interneurons (Gupta et al., 2000)

\footnotetext{
Received July 2, 2002; revised Sept. 6, 2002; accepted Sept. 6, 2002.

This work was supported by the Epilepsy Foundation (M.B), National Institutes of Health Grants GM19214 (C.P.F.), NS40726, and EY11787, and the John Merck Fund (R.Y.). We thank Joshua Brumberg, Barry Connors, Jay Gibson, James Kozloski, Jason McLean, Michael Long, and David Pinto for comments on this manuscript.

Correspondence should be addressed to Dr. Michael Beierlein at his present address: Department of Neurobiology, Harvard Medical School, 220 Longwood Avenue, Boston, MA 02115. E-mail: mbeierlein@hms.harvard.edu. Copyright (C) 2002 Society for Neuroscience $0270-6474 / 02 / 229885-10 \$ 15.00 / 0$
}

that form and receive synapses with distinct short-term properties (Thomson et al., 1996; Gibson et al., 1999; Gupta et al., 2000).

The interaction of recurrent excitation and feedforward and feedback inhibition within layer 4 determines how cortical neurons respond to distinct temporal patterns of TC activity. In vivo studies have shown that neurons in layer 4 are particularly sensitive to the timing of thalamic inputs (Miller et al., 2001). Strong, near synchronous activity among thalamic neurons leads to strong activation of layer 4 neurons, whereas slow changes in thalamic firing evoke only weak responses (Pinto et al., 2002).

Understanding how cortical activity patterns are generated and modified requires data about the spatial patterns of activity, as well as single-cell data within the active network. Here we used calcium imaging to visualize activity in large populations of neurons with single-cell resolution (Yuste and Katz, 1991; Smetters et al., 1999) and whole-cell recording of visually identified neurons to characterize the spatiotemporal activity patterns. Cortical activity was evoked by either low-frequency $(<20 \mathrm{~Hz})$ or short trains of high-frequency $(>40 \mathrm{~Hz})$ TC stimuli. These two frequency regimens approximately reflect the two firing modes of thalamic relay neurons. Tonic, low-frequency firing is typically associated with states of vigilance and attentiveness, whereas high-frequency burst firing is thought to occur mainly during certain sleep states (Steriade, 2000). Burst firing has been also been observed during awake states (Guido and Weyand, 1995) and might carry specific information about sensory stimuli (Reinagel et al., 1999).

We found that, during low-frequency TC activity, only a small number of excitatory neurons fire single action potentials. These neurons were located within small clusters of layer 4, comprised both spiny stellate and star pyramid cells, and fired spikes mediated by activation of only a small number of TC axons. In contrast, high-frequency trains triggered activity in a large num- 
A

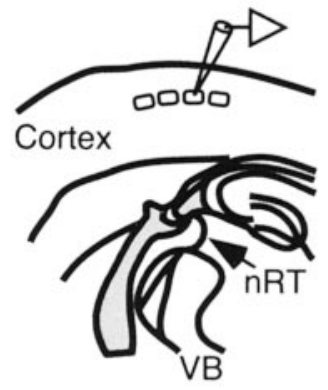

in TC slices. $A$ Schematic of TC slice, with location of approximate recording site in layer 4. $B$, Neocortical layer 4 viewed with $20 \times$ objective. Slice was loaded with fura-2 AM. Outlined area is shown at higher magnification in the right panel. $C, D$, Single TC stimulus evokes response in single neuron. $C$, Normalized fluorescence change for neuron (solid line) shown in $B$ and $D$ and entire field of view (dotted line). Vertical arrow in this and all subsequent figures indicates time of TC stimulation. $D$, Fluorescence change is limited to single neuron. Shown is one frame $(320 \mathrm{msec}$ duration) of $\Delta F / F_{0}$ movie, corresponding to time after the stimulus. Activated neuron is marked by an arrow in both $B$ and $D$.
C

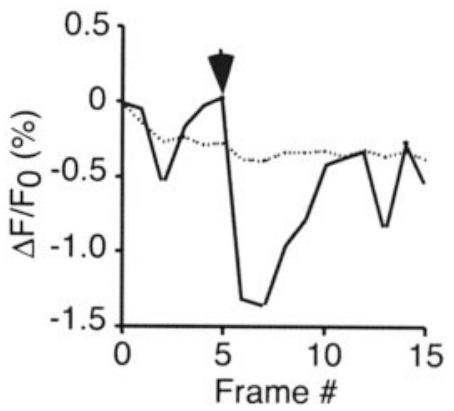

B

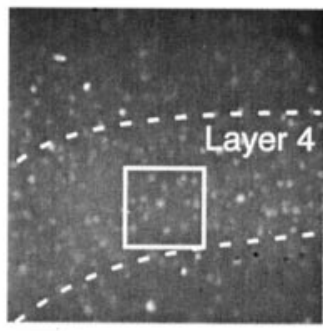

$\overline{100 \mu \mathrm{m}}$

D

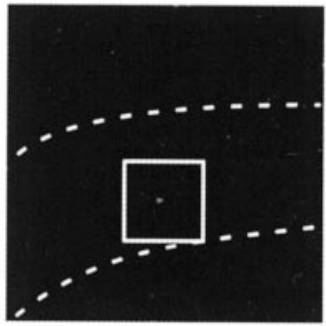

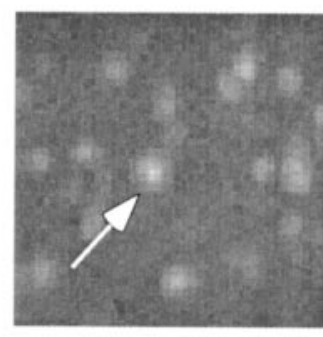

$25 \mu \mathrm{m}$

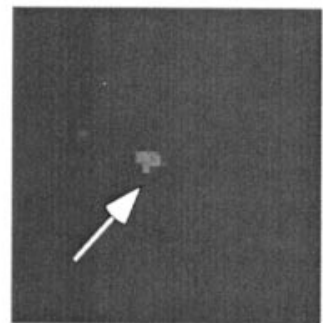

ber of neurons in layer 4, mediated predominantly by intracortical connections, and this activity spread to other neocortical layers. Thus, cortical circuits are particularly sensitive to high-frequency activity in TC neurons.

\section{MATERIALS AND METHODS}

Slice preparation and fura loading. Experiments were performed in accordance with the NIH Guide for the Care and Use of Laboratory Animals (National Institutes of Health publication number 86-23, revised in 1987). Thalamocortical slices, $400 \mu \mathrm{m}$ thick, were obtained from postnatal day 8 (P8) to P16 C57BL/6 mice as described previously (Agmon and Connors, 1991). Animals were anesthetized with $120 \mathrm{mg} / \mathrm{kg}$ ketamine $-10 \mathrm{mg} / \mathrm{kg}$ xylazine and decapitated. The brain was quickly removed and placed into cold artificial CSF (ASCF). Slices were cut with a vibratome (VT1000S; Leica, Nussloch, Germany) and then incubated at $32^{\circ} \mathrm{C}$ for $45 \mathrm{~min}$. The bathing solution contained the following (in $\mathrm{mm}$ ): $126 \mathrm{NaCl}, 3 \mathrm{KCl}, 1.25 \mathrm{NaH}_{2} \mathrm{PO}_{4}, 26 \mathrm{NaHCO}_{3}, 10$ dextrose, $1.3 \mathrm{MgSO}_{4}$, and $2.5 \mathrm{CaCl}_{2}$ (saturated with $95 \% \mathrm{O}_{2}-5 \% \mathrm{CO}_{2}$ ). Some experiments were performed with ACSF containing $2 \mathrm{~mm} \mathrm{MgSO}_{4}$ and $2 \mathrm{mM} \mathrm{CaCl}_{2}$ and led to similar results.

For imaging, slices were loaded with the $\mathrm{Ca}^{2+}$ indicator fura-2 AM (Molecular Probes, Eugene, OR) using a protocol modified by that of Peterlin et al. (2000). Slices were transferred to a small Petri dish filled with 2-3 ml of ACSF aspirated with $95 \% \mathrm{O}_{2}-5 \% \mathrm{CO}_{2}$. An aliquot of 50 $\mu \mathrm{g}$ of fura-2 AM, dissolved in $7 \mu \mathrm{l}$ of DMSO and $2 \mu \mathrm{l}$ of Pluronic (Molecular Probes), was added to the bath for a final concentration of 20 $\mu \mathrm{M}$ fura-2 AM. Slices were incubated for $20 \mathrm{~min}$ at $32^{\circ} \mathrm{C}$, before being transferred into a holding chamber held at room temperature. All experiments were performed at $32^{\circ} \mathrm{C}$.

Imaging. Fura-2 AM responses to changes in intracellular $\mathrm{Ca}^{2+}$ were visualized with an upright fluorescence microscope (Olympus BX50W I; Olympus Optical, Tokyo, Japan) using a $380 \mathrm{~nm}$ excitation filter, a 395 $\mathrm{nm}$ dichroic mirror, and a $510 \mathrm{~nm}$ emission filter (Chroma Technology, Brattleboro, VT). Objectives used included a $20 \times / 0.5$ numerical aperture (NA), a $40 \times / 0.8 \mathrm{NA}$ (water immersion), and a $4 \times / 0.1 \mathrm{NA}$ (air; Olympus Optical). Fluorescent images were taken with a cooled CCD camera (Micromax; Princeton Instruments, Trenton, NJ) equipped with a frametransfer chip (EEV 512). For most experiments, short stimulus-triggered movies of $<6 \mathrm{sec}$ were acquired using IPLAB software (Scanalytics, Vienna, VA). Typically, either $2 \times 2$ or $4 \times 4$ pixels were binned before readout. Camera acquisition was 200 or $320 \mathrm{msec}$ per frame, and each pixel was digitized at 12 bits.
Image analysis. Online image analysis was carried using ImageJ (Wayne Rasband, National Institutes of Health, Bethesda, MD). For each pixel, we measured the fluorescence change (in percentage) over time as $\Delta F / F_{0}=\left(F_{\mathrm{X}}-F_{0}\right) / F_{0}$, where $F_{\mathrm{X}}$ is absolute fluorescence in frame $\mathrm{x}$, and $F_{0}$ denotes fluorescence in the initial frame, before stimulation. The resulting $\Delta F / F_{0}$ movies were used to guide whole-cell recordings of active neurons. Colorized images shown were first filtered with a twopixel radius mean filter to reduce noise. Images were then colorized using a look up table with lighter tones equaling higher $\Delta F / F_{0}$ values. Contrast and gain were adjusted for best visibility. All frames in a sequence or frames comparing two conditions were colorized using the same contrast-gain settings.

Electrophysiology. Thalamocortical fibers were stimulated using bipolar platinum-iridium electrodes (Frederick Haer Co., Bowdoinham, ME) placed in the ventrobasal nucleus (VB) of the thalamus near the reticular nucleus (nRT) (Fig. 1A). In most experiments, slices were placed in the recording chamber with the anterior surface up because complete TC fibers are thought to be close to the anterior surface (Agmon et al., 1993). Stimulus duration ranged from 100 to $200 \mu \mathrm{sec}$, and stimulus intensity ranged from 5 to $120 \mu \mathrm{A}$. In some experiments, the NMDA receptor antagonist APV (Sigma, St. Louis, MO) and the $\mathrm{GABA}_{\mathrm{A}}$ receptor antagonist bicuculline methiodide (Sigma) were used. Whole-cell current-clamp recordings [BVC-700 (Dagan, Minneapolis, MN) and Axoclamp 2B (Axon Instruments, Foster City, CA)] were performed from neurons in layer 4 using 6-8 $\mathrm{M} \Omega$ micropipettes, filled with the following (in $\mathrm{mm}$ ): $136 \mathrm{~K}$-methylsulfate, $2 \mathrm{MgCl}_{2}$, 0.6 EGTA, $10 \mathrm{HEPES}$, 4 ATP-Mg, and 0.3 GTP-Tris, pH 7.2 (295 mOsm). In some experiments, the fluorescent dye Alexa-488 (50 $\mu \mathrm{M}$; Molecular Probes) was added to the internal solution. Responses were digitized at $20 \mathrm{kHz}$ with an analogto-digital board (InstruTech, Minneola, NY), stored using SUPERSCOPE (GW Instruments, Somerville, MA), and analyzed offline with IGOR-Pro software (WaveMetrics, Lake Oswego, OR). Data are expressed as mean $\pm \mathrm{SD}$.

\section{RESULTS}

We loaded TC slices with fura-2 AM and imaged the spatiotemporal activity patterns in layer 4 evoked by thalamic stimulation (Fig. $1 B-D$ ). Barrels were readily identified as regions of high cell density separated by low-density boundaries. Using a $20 \times$ lens, action potential activity could be optically detected in individual neurons in single trials, over a field of one to two adjacent barrel 
columns (Fig. 1C,D). Under our conditions, $80 \pm 15$ layer 4 neurons ( $n=23$ slices) per barrel were loaded well enough to allow for the detection of spike-triggered accumulations in intracellular free calcium concentration $\left(\left[\mathrm{Ca}^{2+}\right]_{i}\right)$.

\section{TC EPSPs evoke action potential firing in excitatory neurons}

Spontaneous activity in layer 4 was low under our conditions. We first measured activity evoked by low-frequency stimulation $(<0.1$ $\mathrm{Hz}$ ). In most cases (28 of 43 slices), no activity in individual neurons in the plane of focus could be detected, regardless of stimulation intensity. In a smaller number of cases (15 of 43 slices), single stimuli triggered activity in one to three neurons (Fig. $1 C, D)$. Single-cell fluorescence transients were small $\left(\Delta F / F_{0}\right.$ $=0.4 \pm 0.08 \% ; n=10)$, suggesting that only few, or even one, action potentials were evoked (Smetters et al., 1999).

To confirm that neuronal $\mathrm{Ca}^{2+}$ transients measured optically were indeed triggered by action potentials, active neurons identified via their somatic $\mathrm{Ca}^{2+}$ transient were recorded with wholecell pipettes (Fig. 2). Some cells were filled with $50 \mu \mathrm{M}$ Alexa to reveal their morphology ( $n=5$; data not shown). Neurons belonged to either one or two morphological categories described previously (Lorente de Nó, 1922; Feldmeyer et al., 1999). Spiny stellate cells had dendritic and axonal arbors confined to a single barrel (data not shown). Cells near the barrel border formed highly asymmetric dendritic and axonal trees. In contrast, star pyramidal neurons had a clear apical dendrite with a distal tuft in layers 1 or 2 (data not shown). We tested the intrinsic firing patterns by applying DC current steps. All neurons were regular spiking (RS) $(n=14)$ (McCormick et al., 1985), with either fast (Fig. $2 A)(n=7)$ or slow (Fig. $2 B)(n=7)$ degree of spike frequency adaptation (Feldmeyer et al., 1999).

All active neurons received strong TC EPSPs that had short and constant trial-to-trial latencies $(3.9 \pm 0.5 \mathrm{msec} ; n=14)$. We measured single-axon EPSPs by adjusting stimulus intensity so that synaptic responses were evoked in only $\sim 50 \%$ of all trials [minimal stimulation (Raastad, 1995; Gil et al., 1999)]. Under these conditions, average EPSPs amplitudes were $9.8 \pm 4.8 \mathrm{mV}$ $(n=10)$. In some neurons, even minimal stimulation led to the generation of action potentials (Fig. 2C). By increasing stimulus intensity, single action potentials could be evoked in every neuron tested. Simultaneous imaging and whole-cell recording confirmed that somatic $\mathrm{Ca}^{2+}$ transients measured optically were indeed triggered by action potentials (data not shown). Spikes were clearly evoked by TC EPSPs because little or no polysynaptic activity was detected. Spike latency fluctuated considerably from trial to trial (Fig. 2D) and was quite variable between neurons (Fig. 3D) (median latency range of 9.2-32 msec; mean of $12.5 \pm$ $5.3 \mathrm{msec} ; n=14$ cells). Latency was strongly influenced by changes in the holding potential. When cells were hyperpolarized by $\sim 5 \mathrm{mV}$, spike latencies increased considerably (Fig. 2E), because many cells generated long-lasting plateau potentials before spike generation (Fricker and Miles, 2000). To estimate how many activated TC fibers contributed to the EPSP amplitude necessary for spike generation, we measured the distribution of evoked EPSP amplitudes by slowly increasing stimulus intensity (Chen and Regehr, 2000). Typically, only one to three step-like increases in amplitude were observed (Fig. $2 F$ ). Thus, convergent input of only a small number of TC neurons evoked action potentials in these neurons.

Because of the small $\mathrm{Ca}^{2+}$ signals evoked by single action potentials, the number of activated neurons might have been
A

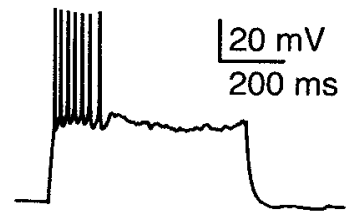

B

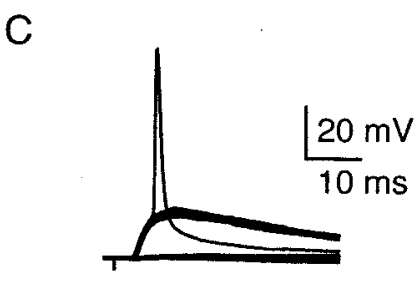

$E$

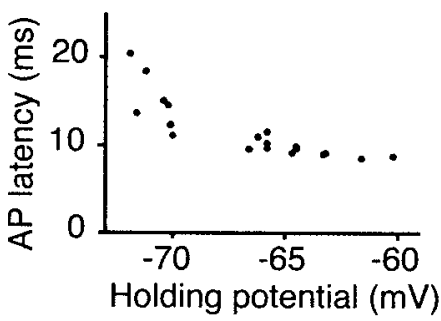

$\mathrm{F}$
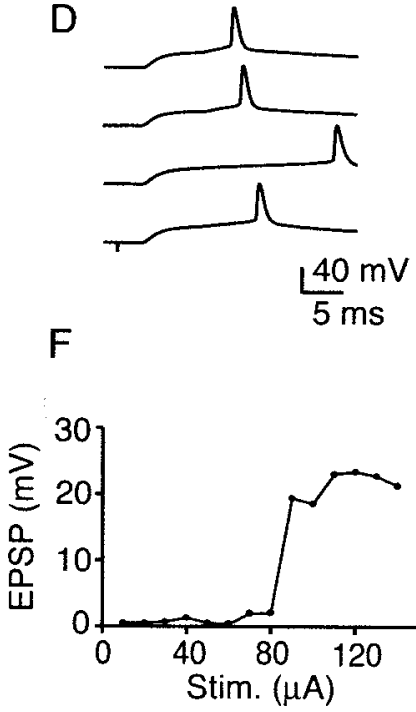

Figure 2. TC EPSPs trigger spikes in layer 4 excitatory neurons. All data except $B$ recorded from neuron shown in Figure 1. $A, B$, Intrinsic firing pattern of RS neurons in layer 4. Depolarizing current steps $(0.1 \mathrm{nA})$ evoked spike firing with either fast $(A)$ or slow $(B)$ degree of spike adaptation. $C$, TC responses in RS cell identified via $\mathrm{Ca}^{2+}$ signal. Stimulus amplitude was adjusted to evoke threshold responses, with an approximately equal number of successes and failures. Multiple trials evoked at $0.1 \mathrm{~Hz}$ are superimposed. $D$, Spike latency jitter in an RS cell. Shown are four consecutive trials evoked at $0.1 \mathrm{~Hz}$. E, Dependence of spike latency on membrane potential in an RS cell. Holding potential was adjusted by small depolarizing or hyperpolarizing currents $(<20 \mathrm{pA})$. $A P$, Action potential. $F$, Small number of TC axons mediates spiking. Incrementing stimulus intensity results in stepwise increase in EPSP amplitude. Cell was hyperpolarized by $\sim 5 \mathrm{mV}$ to prevent spiking.

underestimated. We therefore also performed recordings from RS cells in the same barrel for which no evoked $\mathrm{Ca}^{2+}$ transient could be detected $(n=55)$. Most of these neurons exhibited TC EPSPs (41 of 55 cells), but no action potential firing could be evoked, regardless of stimulus intensity. When tested, single-axon EPSP amplitude in these cells was $5.5 \pm 3.6 \mathrm{mV}(n=11)$, significantly smaller compared with single-axon EPSPs in spikegenerating neurons.

Spike generation in neurons could be aided by their electrotonic compactness. However, input resistance among neurons generating spikes was not significantly higher compared with nonspiking neurons $[577 \pm 176 \mathrm{M} \Omega(n=13)$ vs $484 \pm 188 \mathrm{M} \Omega$ $(n=53) ; p>0.1]$.

In summary, we found that low-frequency TC inputs evoked single spikes in a small group of layer 4 excitatory neurons, mediated by very powerful synapses.

\section{Activation of interneurons by TC axons}

Why is only a minority of layer 4 neurons activated to spike by thalamic stimulation? Previous studies have suggested that TCevoked activity is tightly controlled by feedforward inhibition 
A

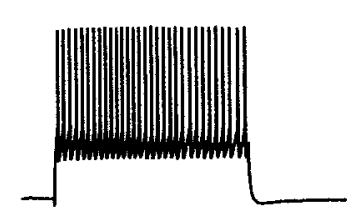

B
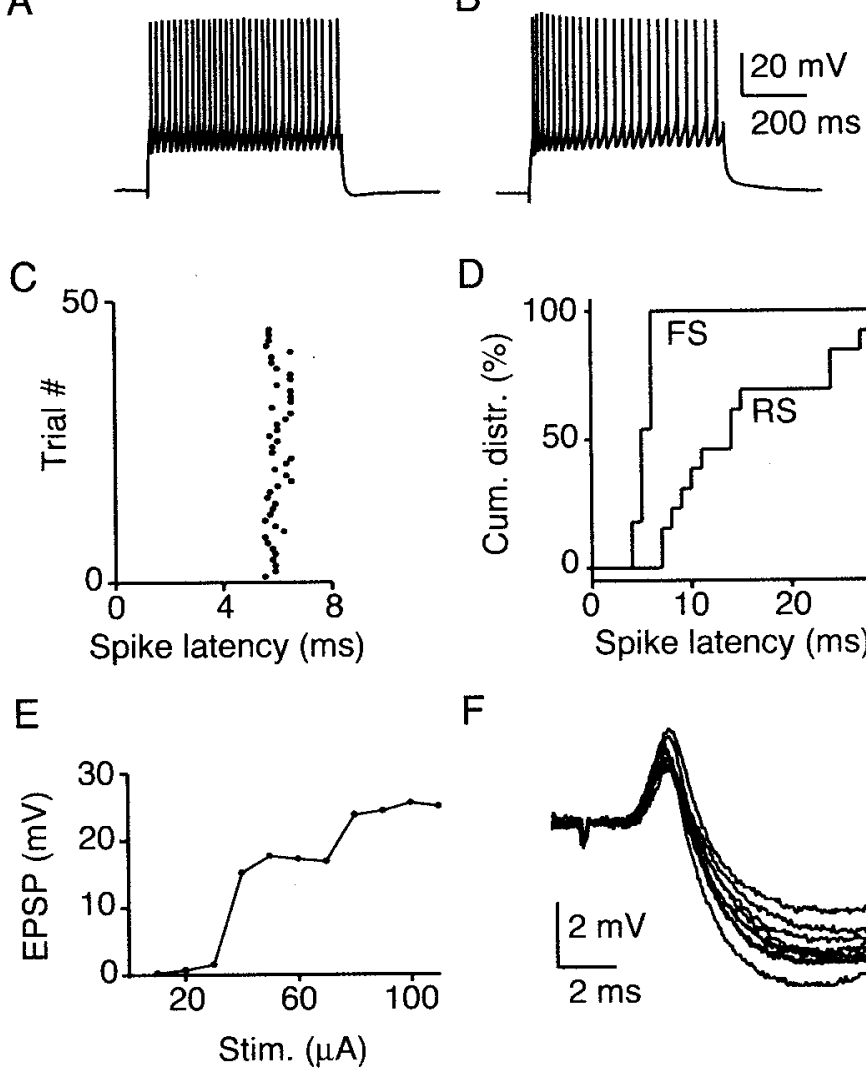

$\mathrm{F}$
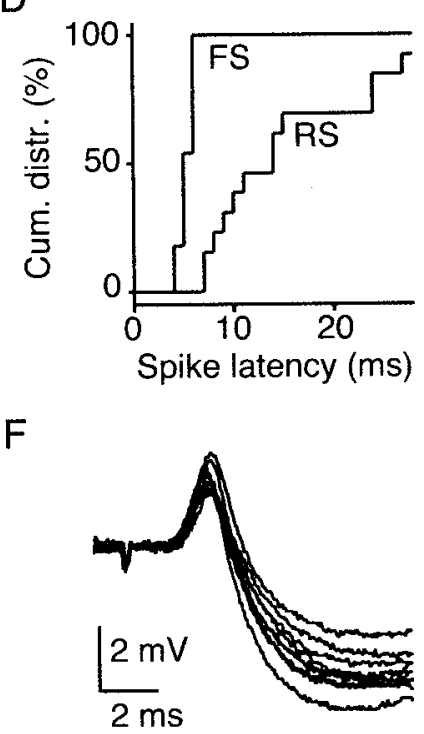

Figure 3. Rapid feedforward inhibition is mediated by FS interneurons. $A, B$, Intrinsic firing in two types of interneurons evoked by depolarizing current steps $(0.2 \mathrm{pA})$. $A$, FS cell, with no spike frequency adaptation. $B$, RSNP cell with moderate spike frequency adaptation. $C$, Precise spike generation in FS neurons triggered by TC EPSPs. Raster plot indicates time of action potential firing in consecutive trials $(0.1 \mathrm{~Hz})$ evoked by single TC stimuli applied at time 0.D, Distribution of spike latencies for FS and RS cells, evoked by single TC EPSPs. Shown are the median values for each cell. $E$, Small number of TC axons evokes spikes in FS neuron. Incrementing stimulus intensity results in stepwise increase in EPSP amplitude. Cell was hyperpolarized by $\sim 5 \mathrm{mV}$ to prevent spiking. $F$, Feedforward inhibition curtails TC EPSPs. Recording from an RS cell, held at $-55 \mathrm{mV}$. Multiple trials are overlaid.

(Gibson et al., 1999; Porter et al., 2001). We recorded from interneurons in the same barrel $(n=42$ cells) and tested whether TC EPSPs could evoke action potentials at latencies fast enough to prevent spike generation in excitatory neurons. Targeted neurons typically had elongated cell bodies and two vertically oriented dendritic processes that originated on opposite sides of the cell body. When filled with Alexa, the dendrites were found to be aspiny or sparsely spiny $(n=4)$, with vertically oriented dendritic trees.

We classified interneurons based on their firing pattern in response to depolarizing current steps. Interneurons could be distinguished from excitatory neurons by their fast $[0.5 \pm 0.2$ $\operatorname{msec}(n=12)$ vs $1.1 \pm 0.2 \operatorname{msec}(n=21)$ in $\mathrm{RS} ; p<0.01]$ undershooting action potentials (McCormick et al., 1985; Kawaguchi, 1995; Gibson et al., 1999; Porter et al., 2001). Following the classification established by Kawaguchi (1995), we used the degree of spike frequency adaptation to distinguish between fast-spiking (FS) neurons $(n=28)$ (Fig. $3 A)$ and regular-spiking nonpyramidal (RSNP) neurons $(n=14)$ (Fig. $3 B)$. None of the cells generated rebound burst firing in re- sponse to hyperpolarizing current steps, as reported for certain interneuron types in other studies (Gibson et al., 1999; Gupta et al., 2000).

Most FS cells received thalamic input ( 25 of 28 cells). The TC EPSP latency $(3.5 \pm 0.9 \mathrm{msec} ; n=25)$ and time-to-peak $(6.2 \pm 1.3 \mathrm{msec})$ were significantly shorter than in RS cells $[4.0 \pm 1.2 \mathrm{msec}(n=55)$ and $10.8 \pm 2.5 \mathrm{msec}(n=46) ; p<$ 0.001]. Single-axon EPSPs were large $(11.9 \pm 5.8 \mathrm{mV} ; n=15)$ and showed little variability in amplitude from trial to trial. Many FS cells generated single action potentials in response to thalamic stimulation (11 of 28 cells). Spikes occurred at short latencies (Fig. $3 D)(5.9 \pm 0.6 \mathrm{msec} ; n=11)$ in all neurons, with little trial-to-trial variability in individual neurons (Fig. 3C). Similar to spike-generating RS cells, spiking in FS cells was mediated by only a small number of TC axons (Fig. 3E). Among RSNP cells, 6 of 14 received thalamic input (threshold EPSP amplitude, $10.6 \pm 3.4 \mathrm{mV} ; n=4)$, and two cells generated action potentials.

The difference in spike latency between FS and RS neurons suggest that FS interneurons can rapidly curtail TC inputs in neighboring excitatory cells. Indeed, when RS neurons were recorded and held at depolarized membrane potentials, rapid and reliable disynaptic inhibition could be observed (Fig. $3 F$ ).

In summary, TC EPSPs led to rapid and reliable spike generation in a large fraction of FS cells, suggesting that low-frequency TC inputs are strongly controlled by feedforward inhibition.

\section{High-frequency stimulus trains trigger recurrent activity}

We then tested cortical activity evoked by brief high-frequency stimulus trains. In contrast to single shocks, stimulus trains (two to six stimuli; 40-100 $\mathrm{Hz}$ ) reliably triggered widespread and longer-lasting activity in large populations of neurons. When measured optically, this "network activity" initiated in small groups of layer 4 neurons before recruiting additional neurons within layer 4 and spreading into layers $2 / 3$ and 5 (Figs. $4 A, 5 A$ ). We used a wide-field objective to estimate the spatial extent of network activity (Fig. $4 B$ ). Activity propagated laterally within layers $2 / 3$ over distances up to $1 \mathrm{~mm}$, whereas spread within layer 4 was restricted to one or two barrels. Network activity had a distinct threshold (Fig. 5A) (see below), and its initiation was dependent on both stimulus frequency and number. Brief $40 \mathrm{~Hz}$ trains (stimulus number was less than six) reliably evoked population activity (95\%; 56 of 59 slices). The likelihood progressively dropped for trains at $20 \mathrm{~Hz}(39 \%), 10 \mathrm{~Hz}(14 \%)$, and single stimuli $(6 \%)$.

To further characterize the properties of network activity, we performed whole-cell recordings from layer 4 neurons (Fig. 4C). Low-frequency stimulus trains evoked monosynaptic, depressing EPSPs, consistent with previous studies (Gil et al., 1997; Gibson et al., 1999). When stimulus frequency was increased beyond threshold, monosynaptic TC activity was followed by long-latency polysynaptic activity. Simultaneous $\mathrm{Ca}^{2+}$ imaging and whole-cell recordings showed that widespread network activity, measured optically, was always correlated with long-latency polysynaptic activity. Long-latency activity was not mediated by temporal summation of TC EPSPs because it was also observed in neurons that did not receive TC inputs (Fig. 4C). At threshold, polysynaptic activity had a latency of $100 \pm 63 \mathrm{msec}(n=59)$ and a duration of $200 \pm 78 \mathrm{msec}(n=59)$. Polysynaptic activity never occurred spontaneously. In 38 of 59 neurons, spikes were triggered during polysynaptic activity (mean, 2.9 spikes; $n=38$ ). 
A

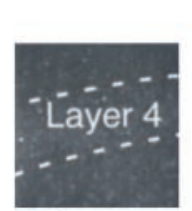

1

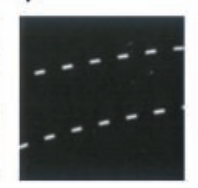

2

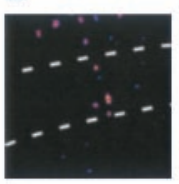

3

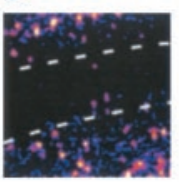

$\overline{100} \mu \mathrm{m}$

B

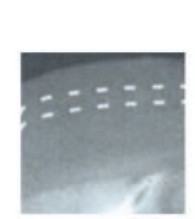

1

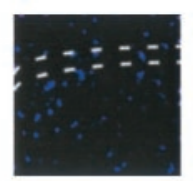

2

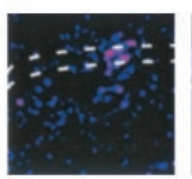

3

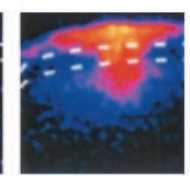

$\overline{500} \mu \mathrm{m}$

C

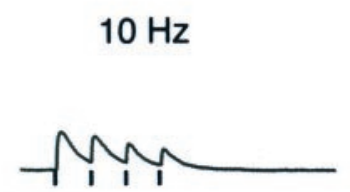

Cell 1
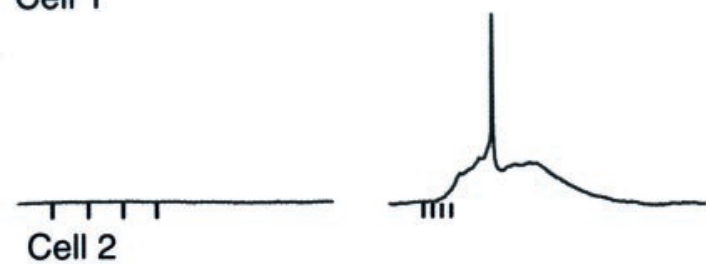

D
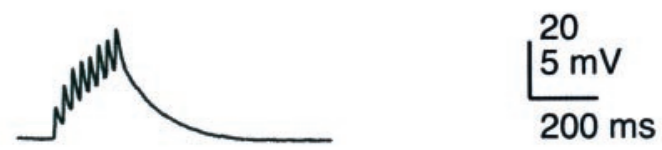

Figure 4. High-frequency bursts trigger recurrent activity. $A$, Network activity in layer 4 evoked by short train of TC stimuli ( 5 stimuli, $40 \mathrm{~Hz}$ ). Leftmost frame shows imaged area in layer 4 , viewed by $20 \times$ objective. Three consecutive frames of $\Delta F / F_{0}$ movie (320 msec duration per frame), with frame 1 preceding the stimulus burst and frames 2 and 3 following the stimulus burst. $B$, Imaging lateral spread of network activity at low magnification. Slice viewed with $4 \times$ objective. Network activity evoked by three stimuli, $40 \mathrm{~Hz}$. Three consecutive frames of $\Delta F / F_{0}$ movie $(200 \mathrm{msec}$ duration per frame), with frame 1 preceding the stimulus burst and frames 2 and 3 following the stimulus burst. Initiation of activity in layer 4 ( frame 2 ) is followed by lateral spread in layers $2 / 3$ and 5 and restricted spread in layer 4 (frame 3). $C$, Network activity is mediated by intracortical polysynaptic activity. Recordings show synaptic responses from two RS neurons in the same barrel, evoked by four stimuli at $10 \mathrm{~Hz}$ (left) and 40 $\mathrm{Hz}($ right $)$. Cell 2 did not receive TC EPSPs but was recruited by polysynaptic activity. $D$, Facilitating monosynaptic EPSP in an FS cell, evoked by thalamic stimulation (8 stimuli, $40 \mathrm{~Hz}$ ). Note the absence of recurrent activity.

When more than one spike was generated, the firing frequency was $43 \pm 25 \mathrm{~Hz}(n=29)$. Excitatory $(n=41)$ as well as both types of inhibitory $(n=19)$ neurons participated in polysynaptic activity, with no significant differences in latency, duration, or amplitude.

In summary, brief high-frequency TC inputs triggered longlatency, polysynaptic activity in both excitatory and inhibitory neurons, which initiated in layer 4 before spreading into layers $2 / 3$ and 5. In the following, we will refer to this type of activity pattern as "recurrent activity."
A

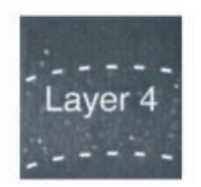

1

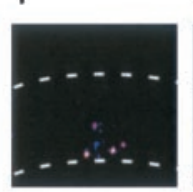

2

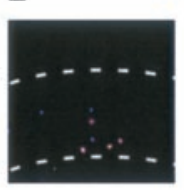

3

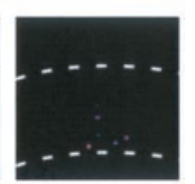

4 pulses

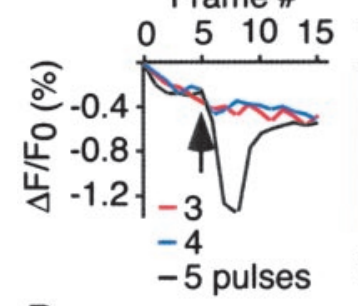

1

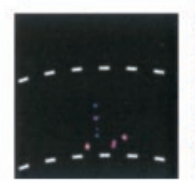

2

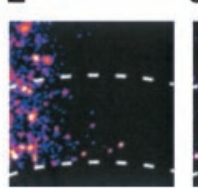

3

5 pulses

B

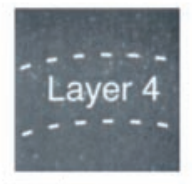

$\overline{100} \mu \mathrm{m}$

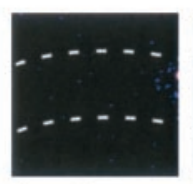

4 pulses

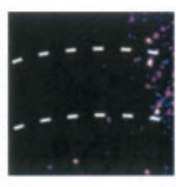

5

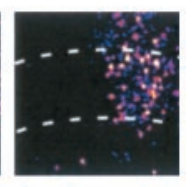

6
Figure 5. Initiation of recurrent activity in layer 4. A, Leftmost frame shows imaged area in layer 4 . Activity evoked by $40 \mathrm{~Hz}$ stimulus train of four (top row) or five pulses (bottom row). Three consecutive frames of $\Delta F / F_{0}$ movie (320 msec duration) are shown for both stimulus trains. Notice that the number and spatial extent of initially activated neurons ( frame 1) are similar, whereas only longer trains evoke recurrent activity (frames 2 and 3, bottom row). Graph plots normalized fluorescence change in imaged area during entire movie, evoked by $40 \mathrm{~Hz}$ trains of three, four, or five pulses. Arrow marks onset of stimulus train. $B$, Stimulus dependence of recurrent activity. Increasing number of pulses beyond the threshold of recurrent activity recruits additional neurons. Shown are three frames (320 msec duration) of three separate $\Delta F / F_{0}$ movies corresponding to the time frame after the respective stimulus train at $40 \mathrm{~Hz}$ (left, 3 pulses; middle, 4 pulses; right, 5 pulses). Initiation site of population activity is to the left of imaged area.

\section{Recurrent activity is triggered by TC synapses and is mediated by intracortical circuits}

The above data indicate that recurrent activity was triggered by TC EPSPs. However, monosynaptic EPSPs could also arise from the antidromic activation of corticothalamic neurons in layer 6 . These neurons form intracortical collaterals that synapse on layer 4 neurons within the same cortical column (Gilbert and Wiesel, 1979; Martin and Whitteridge, 1984; Katz, 1987; Stratford et al., 1996; Zhang and Deschenes, 1997). Antidromically evoked EPSPs typically have longer latencies because of the slower conduction speed of corticothalamic axons (Swadlow, 1990) and display short-term facilitation (Ferster and Lindstrom, 1985) at frequencies that trigger recurrent activity under our conditions. However, all monosynaptic EPSPs ( $n=87$ of 87,59 slices) in slices in which recurrent activity was evoked displayed short-term depression. In a small number of cases ( $n=6$ cells, 6 slices) monosynaptic facilitating responses could be recorded (Fig. 4D), with latencies slightly longer compared with the ones of TC EPSPs $[5.8 \pm 0.9$ $\operatorname{msec}(n=6)$ vs $3.9 \pm 1.2 \operatorname{msec}(n=86) ; p<0.001]$. In those slices, no recurrent activity could be evoked. Thus, recurrent activity was likely triggered by TC inputs with little or no contribution by intracortical synapses of corticothalamic neurons in layer 6 .

It is possible that recurrent activity was initiated in the thalamus and then relayed to cortex. To test this, we performed 
whole-cell recordings from neurons in $\mathrm{nRT}$ and VB and measured their synaptic responses evoked by thalamic stimuli that triggered recurrent activity in cortex. Although thalamic neurons displayed monosynaptic facilitating EPSPs to high-frequency stimulation as shown previously (Turner and Salt, 1998), no polysynaptic activity could be detected. These data suggest that recurrent activity was generated within cortical circuits.

\section{Recurrent activity is not developmentally regulated and can be evoked in unloaded slices}

The above data indicate that high-frequency inputs can overcome feedforward inhibition and trigger long-lasting activity in a large number of excitatory and inhibitory neurons. It is possible that recurrent activity could result from immature inhibitory circuitry in very young animals (Agmon et al., 1996). However, we found no correlation between the likelihood of evoking recurrent activity and animal age in slices of animals ranging from P8 to P16.

We were also concerned that fura loading and phototoxicity induced by imaging might have increased the excitability of our slices, thus explaining the generation of recurrent activity. We performed control experiments in unloaded slices and tested whether brief TC stimulus trains led to activity with the same electrophysiological characteristics described above for recurrent activity. Indeed, long-latency polysynaptic activity specifically evoked by high-frequency stimulus trains was observed in four of five experiments (P10-P16).

\section{Threshold initiation of recurrent activity in small clusters of layer 4 neurons}

The above data suggest that short trains of TC EPSPs trigger a small number of excitatory neurons in layer 4 to fire action potentials that then recruit recurrent activity. We estimated the number and spatial extent of active neurons necessary to generate recurrent activity by optically measuring activity patterns just below and above threshold (Fig. 5A). Stimulus parameters were carefully adjusted so that a stimulus train at a given frequency and a given number of pulses would evoke recurrent activity but would fail when the number of pulses was decreased by one. For stimulus trains just below threshold, activity in only a small number (3-10) of neurons was detected. Active neurons were typically localized in layer 4 within areas $\sim 50 \%$ the size of a barrel. Whole-cell recordings revealed that action potentials in these neurons were generated by temporal summation of TC EPSPs (data not shown), with little contribution of long-latency polysynaptic activity.

Stimulus trains just above threshold initiated activity within a cluster of neurons as under subthreshold conditions but then reliably evoked more widespread recurrent activity. These observations suggest that only a small number of neurons, activated by temporally summating TC EPSPs, are sufficient to trigger recurrent activity.

\section{Spatial extent of recurrent activity is controlled by TC stimulus}

We tested whether recurrent activity can be further modulated by the temporal pattern of afferent activity or whether it displays all-or-none behavior. By carefully adjusting stimulus number and intensity, we evoked recurrent activity at threshold. Further increasing the number of pulses in the stimulus train led to recruitment of additional neurons, demonstrating that the spatial extent of recurrent activity is controlled by the temporal pattern of TC activity (Fig. 5B).

Many network phenomena observed in cortex do not share these dynamics. In particular, seizure-like activity induced by application of $\mathrm{GABA}_{\mathrm{A}}$ receptor blockers displays all-or-none behavior. We probed activity in layer 4 after bath applications of low doses of bicuculline (1-5 $\mu \mathrm{M} ; n=5$; data not shown). Under these conditions, thalamic stimuli led to activation of large populations of neurons in all layers, often organized as waves over the entire extent of the slice. Both single stimuli and trains led to seizure-like activity, which was stereotypical in its extent, independent of the temporal pattern of afferent stimulation.

Our data thus indicate that recurrent activity as described above is distinct from seizure-like activity in that it is characterized by graded and spatially restricted neuronal activity, evoked specifically by high-frequency inputs.

\section{Sustaining recurrent activity requires NMDA receptors}

Previous studies have shown that neurons of layer 4 neurons are heavily interconnected by synapses expressing NMDA receptors with only modest voltage dependence (Fleidervish et al., 1998). To probe the robustness of recurrent activity, we imaged responses after bath application of the NMDA receptor antagonist APV at low concentrations $(1-5 \mu \mathrm{M} ; n=9)$. In all cases, recurrent activity was either blocked or its threshold significantly increased (Fig. 6A). Intracellular recordings revealed a progressive delay in the onset of polysynaptic activity followed by complete failure (Fig. 6B), whereas monosynaptic TC activity was unaffected under these conditions. Similarly, the cholinergic agonist muscarine (2-5 $\mu \mathrm{M}$; data not shown), whose action includes a reduction of release probability at excitatory synapses (Gil et al., 1997), blocked recurrent activity (four of four slices).

\section{Linear recruitment of layer $\mathbf{4}$ neurons by TC inputs}

The above data indicate that recurrent activity had a distinct threshold, below which activity was mediated mainly by monosynaptic TC inputs and above which it was mediated by polysynaptic intracortical inputs. Although unlikely, it is possible that this threshold was a reflection of a sudden increase in TC activity, e.g., attributable to the recruitment of TC fibers at high stimulation frequencies. To test this hypothesis, we determined the recruitment of cortical neurons for stimulus trains with an increasing number of pulses in the presence of APV ( $3 \mu \mathrm{M} ; n=5$ slices $)$ to block recurrent activity. In all cases, increases in the number of pulses led to a gradual increase in activity among neurons (Fig. 7 ), most of which were located in layer 4 . These data imply that the threshold of recurrent activity is an emergent property of a highly interconnected network in layer 4.

\section{Recurrent activity can be evoked repetitively}

Intracortical excitatory synapses display use-dependent depression (Thomson and Deuchars, 1994; Gil et al., 1997). Recovery from depression, as well as other factors, will likely control the time interval at which recurrent activity can be evoked successively without decay. We evoked recurrent activity twice, by applying two TC trains at various interburst intervals and characterized the degree of decay for the response evoked by the second burst as a function of interburst interval. Stimulus bursts with intervals as low as $2 \mathrm{sec}$ reliably evoked recurrent activity without decay (mean, $4.2 \pm 1.4 \mathrm{sec} ; n=10$ slices).

When recurrent activity was evoked multiple times at frequencies $>0.2 \mathrm{~Hz}$, a successive decay in the response could be observed (Fig. 8). However, the time course of the decay was different for layer 4 versus layers $2 / 3$ and 5 . Whereas recurrent activity could be evoked reliably in layer 4 with only modest decay 
A

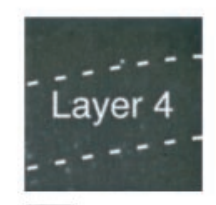

Control
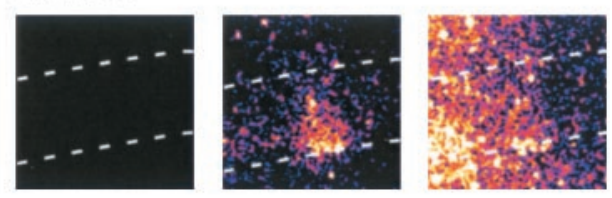

$\overline{100 \mu \mathrm{m}}$

APV
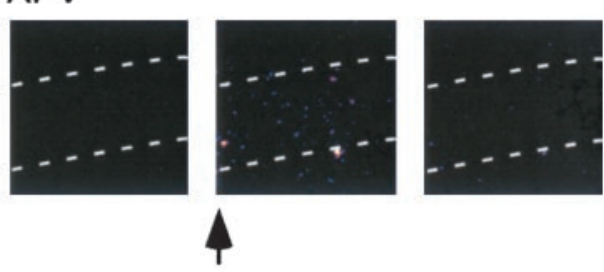

B

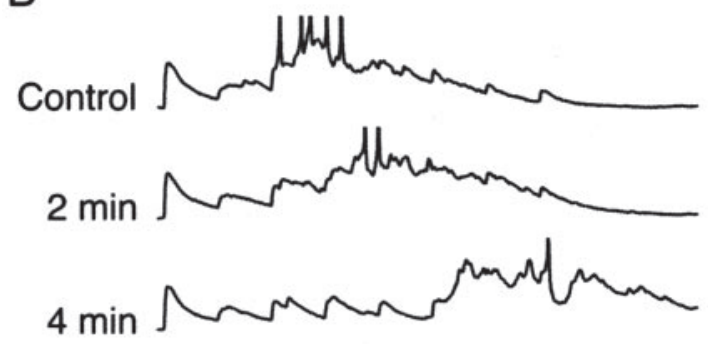

$$
6 \mathrm{~min} \sim \mathrm{N}^{\frac{10 \mathrm{mV}}{50 \mathrm{~ms}}}
$$

Figure 6. Recurrent activity is blocked by reducing NMDA-mediated EPSPs. $A$, Activity evoked by four stimuli at $40 \mathrm{~Hz}$, before (top row) and 5 min after (bottom row) application of $2 \mu \mathrm{M}$ APV . For each case, three consecutive frames of $\Delta F / F_{0}$ movie ( $320 \mathrm{msec}$ duration per frame) are shown, with frame 1 preceding the stimulus burst and frames 2 and 3 following the stimulus burst. $B$, APV completely blocks recurrent activity and slightly attenuates TC EPSPs. Shown are responses in an FS cell, evoked every 2 min by TC stimulus train ( 8 stimuli, $20 \mathrm{~Hz}$ ) after the application of $2 \mu \mathrm{M}$ APV. Notice the increasing delay of recurrent activity before failure. Spikes are truncated.

and variability, activity patterns in layers $2 / 3$ and 5 were much more variable and showed a higher number of failures.

Together, these data suggest that recurrent activity has a relatively short refractory period and can thus be evoked repetitively. However, its spatial extent and onset depend on the recent history of activity.

\section{DISCUSSION}

We combined $\mathrm{Ca}^{2+}$ imaging of large populations of neurons with single-cell resolution with whole-cell recordings to characterize responses in neocortex evoked by different temporal patterns of thalamic stimulation. Low-frequency TC activity led to spike firing in a small subset of layer 4 excitatory neurons, mediated almost exclusively by TC EPSPs. In contrast, brief high-frequency bursts evoked widespread activity in cortex, which initiated in small clusters of neurons in layer 4 , before spreading into layers $2 / 3$ and 5. Previous work has shown that burst firing can increase the reliability of information flow between individual neurons, because of either presynaptic or postsynaptic mechanisms (Lisman, 1997; Swadlow and Gusev, 2001). Our studies show that high-frequency afferent activity can recruit cortical circuits by triggering recurrent excitation within highly interconnected networks of neurons in layer 4.
A

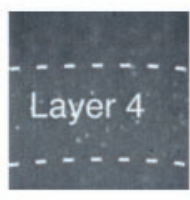

$\overline{100} \mu \mathrm{m}$

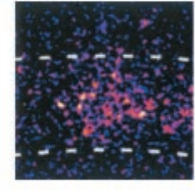

4 pulses

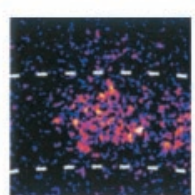

5

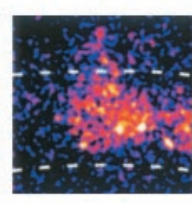

6
B

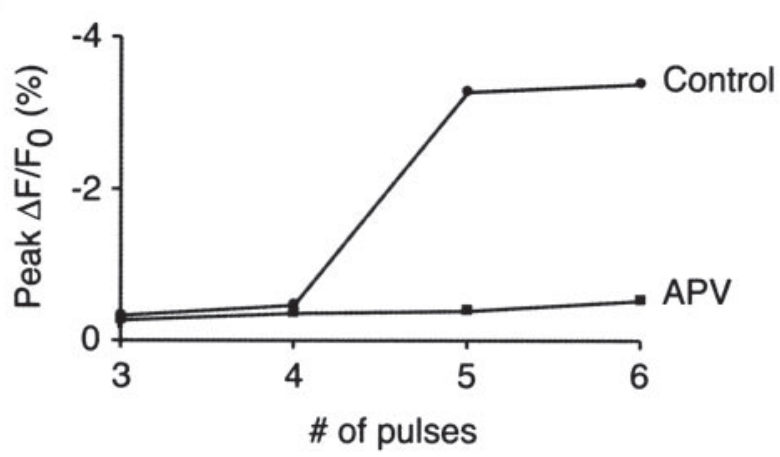

Figure 7. Near-linear recruitment of layer 4 neurons by TC stimulus trains. Activity was evoked in the presence of $2 \mu \mathrm{M}$ APV. Shown are three frames (320 msec duration) of three separate $\Delta F / F_{0}$ movies corresponding to the time frame after the respective stimulus train at $40 \mathrm{~Hz}$ for four, five, and six pulses. Notice the gradual increase of activity, without significant spread into layers $2 / 3$ and 5 . B, Graph plots peak normalized fluorescence change in imaged area, under control conditions and after APV application as shown in $A$, evoked by stimulus trains of three to six pulses at $40 \mathrm{~Hz}$.

\section{Activity evoked by low-frequency stimulation}

A novel finding of our study is the existence of excitatory neurons in layer 4 that fire spikes evoked by only a small number of TC afferents. These neurons likely play an important gating function during the initial processing of sensory inputs in cortex. Our imaging approach allowed us to selectively target these neurons for recordings, via their somatic $\mathrm{Ca}$ transients evoked by action potential firing. Their number appears to be very small, i.e., $<1 \%$ of all excitatory neurons in layer 4. Threshold EPSPs had large amplitudes $(\sim 10 \mathrm{mV})$ in these neurons, likely mediated by a large number of release sites and a high probability of release (Stratford et al., 1996; Gil et al., 1999). Previous work in cat and rat have reported average TC EPSP amplitudes of $\sim 2 \mathrm{mV}$ (Stratford et al., 1996; Gibson et al., 1999), and it seems likely that the neurons described here remained undetected in those studies.

Whereas only a small number of excitatory neurons could be activated by TC EPSPs a significant percentage of interneurons, both FS and RSNP were found to generate TC EPSP-mediated spikes, in agreement with previous studies (Swadlow et al., 1998; Gibson et al., 1999; Porter et al., 2001).

Given the preferential activation of interneurons by TC inputs, a number of studies have concluded that sensory evoked activity in cortex is strongly controlled by feedforward inhibition (Gibson et al., 1999; Porter et al., 2001). Studies in hippocampus (Fricker and Miles, 2000; Pouille and Scanziani, 2001) and neocortex (Galarreta and Hestrin, 2001) have suggested that feedforward inhibition limits the time window in which excitatory inputs can summate to generate action potential firing. Interestingly, spike latency among excitatory neurons in our study was significantly longer than for interneurons. Thus, most neurons generated spikes well after the onset of feedforward inhibition. It is possible 

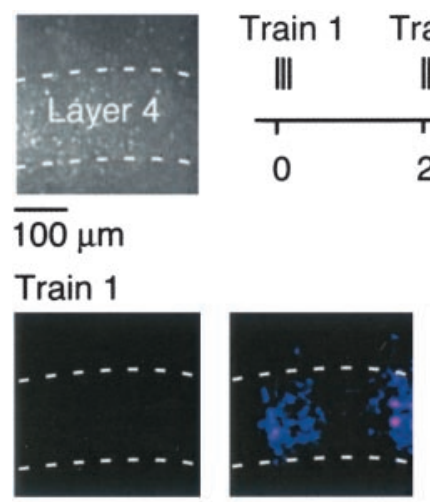

Train 2 Train $3 \quad$ Train 6 $\overline{100} \mu \mathrm{m}$

Train 1
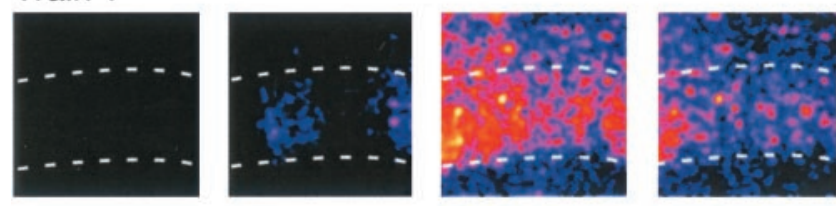

Train 2
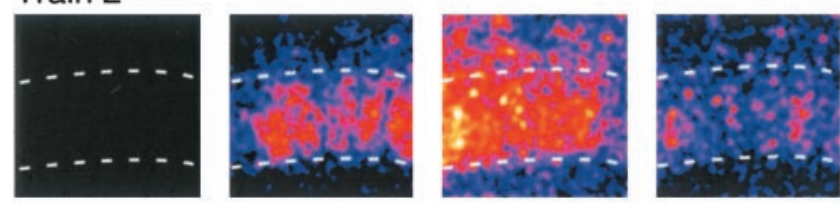

Train 6
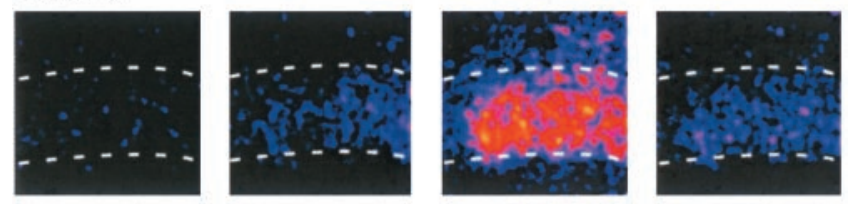

4

Figure 8. Population activity can be evoked repetitively. Recurrent activity was evoked by six consecutive stimulus trains ( 3 stimuli, $40 \mathrm{~Hz}$ ) at $0.5 \mathrm{~Hz}$. Shown are cortical activity patterns after the first, second, and sixth stimulus train. Frame duration, $200 \mathrm{msec}$. Notice the persistence of activity in layer 4 and the larger response variability in layers $2 / 3$.

that, in juvenile (P8-P15) animals, inhibitory synapses are less developed, allowing for a longer time window of synaptic integration in excitatory cells. However, studies in rats (Gibson et al., 1999) and mice (Agmon et al., 1996; Porter et al., 2001) as well as this study suggest the presence of feedforward inhibition even at early developmental stages. Alternatively, it is possible that feedforward inhibition is spatially non-uniform in its impact, allowing a special type of excitatory neuron to temporally summate EPSPs over a longer time window.

\section{Detecting activity in single neurons}

Using a novel imaging approach allowed us to detect single-cell activity within large populations of neurons (Yuste and Katz, 1991; Peterlin et al., 2000). Nevertheless, there are caveats that need to be considered when interpreting our results. It is likely that action potential-evoked activity is underestimated using our approach, especially when neurons only fire single action potentials and thus generate only a small somatic $\mathrm{Ca}^{2+}$ signal. A case in point is the detection of interneuron firing evoked by single thalamic stimuli. Whereas the number of activated interneurons detected optically is comparable with the number of excitatory neurons, electrophysiological methods suggest that their numbers are significantly larger. Single action potential firing might go undetected in interneurons because of their small somatic $\mathrm{Ca}^{2+}$ transients, perhaps the result of a small density of $\mathrm{Ca}^{2+}$ channels or stronger intracellular calcium buffering (J. Goldberg, G. Tamas, and R. Yuste, unpublished observations).

\section{Properties of recurrent activity}

Our main finding is that, whereas low-frequency activity led to spiking in only a small number of excitatory neurons, highfrequency trains of TC inputs inevitably led to widespread activity in layer 4 and in other cortical layers. The strong frequency dependence of recurrent activity suggests that temporal summation of TC inputs is necessary to recruit action potential firing in a sufficiently large number of layer 4 neurons to trigger selfsustaining activity via intracortical synapses.

Multiple mechanisms could explain the propensity of layer 4 to generate recurrent activity. Excitatory neurons within single barrels are strongly interconnected (Feldmeyer et al., 1999; Petersen and Sakmann, 2000), thus favoring the generation of recurrent excitatory loops (Douglas et al., 1995). Furthermore, excitatory synapses between layer 4 neurons might function primarily via NMDA receptors with only modest voltage dependence (Fleidervish et al., 1998), allowing for the generation of persistent activity.

A question unexplored in our study is how ongoing thalamocortical activity and its effect on synaptic strength can influence cortical responses to both low- and high-frequency activity. Recent studies have suggested that the effectiveness of thalamocortical bursts in triggering action potentials in cortical interneurons arises from the sustained period of silence preceding each burst, allowing for significant recovery from short-term depression at thalamocortical synapses (Swadlow and Gusev, 2001) (see also Sherman, 2001). On the other hand, tonic low-frequency activity with on average briefer interspike intervals might be accompanied by a larger degree of steady-state synaptic depression (Chung et al., 2002). Thus, the high responsiveness of cortical circuits to high-frequency thalamocortical activity might be mediated by both presynaptic and postsynaptic mechanisms.

Previous work has shown that the response behavior of cortical neurons is determined by the synaptic properties and connectivity of cortical afferents. Individual thalamic inputs have powerful influence on the response behavior of cortical neurons (Reid and Alonso, 1995; Swadlow and Gusev, 2001). However, few experimental studies have directly examined the influence of intracortical synapses in modulating afferent activity. In the thalamic input layers of cat V1, TC synapses comprise only $\sim 6 \%$ of all excitatory synapses, whereas most inputs are provided by excitatory neurons within the same local circuit (Ahmed et al., 1994). Our study provides insight into the conditions under which these local circuits are recruited and how they influence the response behavior of individual neurons.

\section{Model for the generation of recurrent activity}

Based on the present and previous results, we propose the following model for the generation of recurrent activity. Lowfrequency stimuli trigger activity in a small number of neurons directly activated by TC EPSPs. In contrast, high-frequency TC stimuli leads to temporal summation of EPSPs and thus to the recruitment of both excitatory and inhibitory neurons. Temporal summation of EPSPs is likely to be more effective in excitatory than in inhibitory neurons, because the former have longer membrane time constants, larger NMDA components in their TC EPSP, and possibly different types and distributions of postsynaptic conductances compared with interneurons. Thus, the number of recruited excitatory neurons will likely outpace the number of recruited inhibitory cells. With an increasing number of activated neurons, the contribution of intracortical excitation will 
become more prominent, and, eventually, recurrent activity will be generated.

\section{Comparison with other imaging studies examining TC-evoked activity}

Using $\mathrm{Ca}^{2+}$ imaging in combination with electrophysiology has allowed us to study cortical activity patterns with single-cell resolution. Several recent studies have used voltage-sensitive dyes to characterize activity patterns in response to TC activation ( $\mathrm{Wu}$ et al., 1999; Laaris et al., 2000; Contreras and Llinas, 2001; Petersen and Sakmann, 2001). When comparing our results with those studies, important technical aspects have to be kept in mind. Voltage-sensitive dyes allow for the characterization of network dynamics with very high temporal resolution, but they do not permit the detection of activity in individual neurons. Furthermore, the fluorescent signal measured with voltage-sensitive dyes likely originates from both subthreshold synaptic potentials and action potentials, whereas $\mathrm{Ca}^{2+}$ signals measured in our study are predominantly mediated by somatic action potential firing.

Only Laaris et al. (2000) performed their experiments with a similar stimulus paradigm, i.e., extracellular stimulation in the $\mathrm{VB}$ of the mouse thalamus. In agreement with our results, they showed that single pulses, regardless of intensity, evoked activity mostly in layer 4 of a single barrel column, whereas short highfrequency stimuli recruited widespread activity in supragranular and infragranular layers that was blocked by NMDA receptor antagonists. It is likely that this activity corresponds to the recurrent activity described in our study.

Our results might appear to be in contrast with those obtained in a voltage-sensitive dye study of guinea pig visual and somatosensory cortex (Contreras and Llinas, 2001) that showed that low-frequency stimuli applied to afferent inputs led to widespread activity in layers $2 / 3$ and 5 , whereas high-frequency stimuli limited the lateral spread of activity, because of the recruitment of feedforward inhibition. However, besides differences in species and methodology, cortical activity was evoked by extracellular stimulation in the underlying white matter. It is possible that, in addition to $\mathrm{TC}$ inputs, white matter stimulation led to antidromic recruitment of layers 5 and 6 neurons, which could result in the generation of different spatiotemporal patterns of activation. Direct comparisons between thalamic and white matter stimulation appear necessary.

Recurrent activity as described in our study was initiated within groups of layer 4 neurons, which in turn received strong TC excitation, had a distinct threshold, showed little trial-to-trial variability beyond threshold, and could be controlled in its spatial spread by the stimulation pattern. These properties are in marked contrast to the properties of evoked "population activity" in rat auditory cortex described previously (Metherate and Cruikshank, 1999; Wu et al., 1999). In those studies, population activity could be evoked with a single shock, was typically all-or-none, and could not be controlled by the stimulus parameters.

At this point, it is unclear what explains these discrepancies. It is possible that stimulation site, ionic conditions, or differences between species or cortical areas influence the properties of recurrent activity.

\section{Functional implications}

The recurrent activity that we describe shares some similarities with the reverberating action of neocortical networks proposed in the past (Lorente de Nó, 1938; Hebb, 1949). Specifically, the existence of a form of activity that can persist in the cortex and can outlast thalamic stimulation indicates that the cortical circuit can preserve temporally a record of past activity. This feature is consistent with theories that argue that the cortex, as a heavily interconnected feedback circuit, might operate via attractor dynamics (Hopfield, 1982).

Regardless of its computational significance, we would argue that the recurrent activity as described here appears to be a suitable mechanism to selectively gate sensory information mediated by high-frequency TC activity. Moreover, the dependence of recurrent activity on both temporal pattern of afferent activity as well as its recent history suggests that local cortical networks with a high gain can nevertheless encode complex patterns of sensory information.

\section{REFERENCES}

Agmon A, Connors BW (1991) Thalamocortical responses of mouse somatosensory (barrel) cortex in vitro. Neuroscience 41:365-379.

Agmon A, Yang LT, O'Dowd DK, Jones EG (1993) Organized growth of thalamocortical axons from the deep tier of terminations into layer IV of developing mouse barrel cortex. J Neurosci 13:5365-5382.

Agmon A, Hollrigel G, O’Dowd DK (1996) Functional GABAergic synaptic connection in neonatal mouse barrel cortex. $J$ Neurosci 16:4684-4695.

Ahmed B, Anderson JC, Douglas RJ, Martin KA, Nelson JC (1994) Polyneuronal innervation of spiny stellate neurons in cat visual cortex. J Comp Neurol 341:39-49.

Chen C, Regehr WG (2000) Developmental remodeling of the retinogeniculate synapse. Neuron 28:955-966.

Chung S, Li X, Nelson SB (2002) Short-term depression at thalamocortical synapses contributes to rapid adaptation of cortical sensory responses in vivo. Neuron 34:437-446.

Contreras D, Llinas R (2001) Voltage-sensitive dye imaging of neocortical spatiotemporal dynamics to afferent activation frequency. J Neurosci 21:9403-9413.

Douglas RJ, Koch C, Mahowald M, Martin KA, Suarez HH (1995) Recurrent excitation in neocortical circuits. Science 269:981-985.

Feldmeyer D, Egger V, Lubke J, Sakmann B (1999) Reliable synaptic connections between pairs of excitatory layer 4 neurones within a single "barrel" of developing rat somatosensory cortex. J Physiol (Lond) 521:169-190.

Ferster D, Lindstrom S (1985) Augmenting responses evoked in area 17 of the cat by intracortical axon collaterals of cortico-geniculate cells. J Physiol (Lond) 367:217-232.

Fleidervish IA, Binshtok AM, Gutnick MJ (1998) Functionally distinct NMDA receptors mediate horizontal connectivity within layer 4 of mouse barrel cortex. Neuron 21:1055-1065.

Fricker D, Miles R (2000) EPSP amplification and the precision of spike timing in hippocampal neurons. Neuron 28:559-569.

Galarreta M, Hestrin S (2001) Spike transmission and synchrony detection in networks of GABAergic interneurons. Science 292:2295-2299.

Gibson JR, Beierlein M, Connors BW (1999) Two networks of electrically coupled inhibitory neurons in neocortex. Nature 402:75-79.

Gil Z, Connors BW, Amitai Y (1997) Differential regulation of neocortical synapses by neuromodulators and activity. Neuron 19:679-686.

Gil Z, Connors BW, Amitai Y (1999) Efficacy of thalamocortical and intracortical synaptic connections: quanta, innervation, and reliability. Neuron 23:385-397.

Gilbert CD, Wiesel TN (1979) Morphology and intracortical projections of functionally characterised neurones in the cat visual cortex. Nature 280:120-125

Guido W, Weyand T (1995) Burst responses in thalamic relay cells of the awake behaving cat. J Neurophysiol 74:1782-1786.

Gupta A, Wang Y, Markram H (2000) Organizing principles for a diversity of GABAergic interneurons and synapses in the neocortex. Science 287:273-278.

Hebb DO (1949) The organization of behavior. New York: Wiley.

Hopfield JJ (1982) Neural networks and physical systems with emergent collective computational abilities. Proc Natl Acad Sci USA 79:2554-2558.

Katz LC (1987) Local circuitry of identified projection neurons in cat visual cortex brain slices. J Neurosci 7:1223-1249.

Kawaguchi Y (1995) Physiological subgroups of nonpyramidal cells with specific morphological characteristics in layer II/III of rat frontal cortex. J Neurosci 15:2638-2655.

Laaris N, Keller A (2002) Functional independence of layer IV barrels. J Neurophysiol 87:1028-1034.

Laaris N, Carlson GC, Keller A (2000) Thalamic-evoked synaptic interactions in barrel cortex revealed by optical imaging. J Neurosci 20:1529-1537. 
Lisman JE (1997) Bursts as a unit of neural information: making unreliable synapses reliable. Trends Neurosci 20:38-43.

Lorente de Nó R (1922) La corteza cerebral del ratón. Trabajos del Laboratorio de Investigaciones Biológicas de la Universidad de Madrid 20:41-78.

Lorente de Nó R (1938) Analysis of the activity of the chains of internuncial neurons. J Neurophysiol 1:207-244.

Martin KA, Whitteridge D (1984) Form, function and intracortical projections of spiny neurones in the striate visual cortex of the cat. J Physiol (Lond) 353:463-504.

McCormick DA, Connors BW, Lighthall JW, Prince DA (1985) Comparative electrophysiology of pyramidal and sparsely spiny stellate neurons of the neocortex. J Neurophysiol 54:782-806.

Metherate R, Cruikshank SJ (1999) Thalamocortical inputs trigger a propagating envelope of gamma-band activity in auditory cortex in vitro. Exp Brain Res 126:160-174.

Miller KD, Pinto DJ, Simons DJ (2001) Processing in layer 4 of the neocortical circuit: new insights from visual and somatosensory cortex. Curr Opin Neurobiol 11:488-497.

Mountcastle VB (1998) Perceptual neuroscience: the cerebral cortex. Cambridge, MA: Harvard UP.

Peterlin ZA, Kozloski J, Mao BQ, Tsiola A, Yuste R (2000) Optical probing of neuronal circuits with calcium indicators. Proc Natl Acad Sci USA 97:3619-3624.

Petersen CC, Sakmann B (2000) The excitatory neuronal network of rat layer 4 barrel cortex. J Neurosci 20:7579-7586.

Petersen CC, Sakmann B (2001) Functionally independent columns of rat somatosensory barrel cortex revealed with voltage-sensitive dye imaging. J Neurosci 21:8435-8446.

Pinto DJ, Hartings JA, Brumberg JC, Simons DJ (2002) Cortical damping: Analysis of thalamocortical response transformations in rodent barrel cortex. Cereb Cortex.

Porter JT, Johnson CK, Agmon A (2001) Diverse types of interneurons generate thalamus-evoked feedforward inhibition in the mouse barrel cortex. J Neurosci 21:2699-2710.

Pouille F, Scanziani M (2001) Enforcement of temporal fidelity in pyramidal cells by somatic feed-forward inhibition. Science 293:1159-1163.

Raastad M (1995) Extracellular activation of unitary excitatory synapses between hippocampal CA3 and CA1 pyramidal cells. Eur J Neurosci 7:1882-1888.

Reid RC, Alonso JM (1995) Specificity of monosynaptic connections from thalamus to visual cortex. Nature 378:281-284.
Reinagel P, Godwin D, Sherman SM, Koch C (1999) Encoding of visual information by LGN bursts. J Neurophysiol 81:2558-2569.

Sherman SM (2001) A wake-up call from the thalamus. Nat Neurosci 4:344-346.

Simons DJ (1978) Response properties of vibrissa units in rat SI somatosensory neocortex. J Neurophysiol 41:798-820.

Smetters D, Majewska A, Yuste R (1999) Detecting action potentials in neuronal populations with calcium imaging. Methods 18:215-221.

Steriade M (2000) Corticothalamic resonance, states of vigilance and mentation. Neuroscience 101:243-276.

Stratford KJ, Tarczy-Hornoch K, Martin KA, Bannister NJ, Jack JJ (1996) Excitatory synaptic inputs to spiny stellate cells in cat visual cortex. Nature 382:258-261.

Swadlow HA (1990) Efferent neurons and suspected interneurons in S-1 forelimb representation of the awake rabbit: receptive fields and axonal properties. J Neurophysiol 63:1477-1498.

Swadlow HA, Gusev AG (2001) The impact of "bursting" thalamic impulses at a neocortical synapse. Nat Neurosci 4:402-408.

Swadlow HA, Beloozerova IN, Sirota MG (1998) Sharp, local synchrony among putative feed-forward inhibitory interneurons of rabbit somatosensory cortex. J Neurophysiol 79:567-582.

Thomson AM, Deuchars J (1994) Temporal and spatial properties of local circuits in neocortex. Trends Neurosci 17:119-126.

Thomson AM, West DC, Hahn J, Deuchars J (1996) Single axon IPSPs elicited in pyramidal cells by three classes of interneurones in slices of rat neocortex. J Physiol (Lond) 496:81-102.

Turner JP, Salt TE (1998) Characterization of sensory and corticothalamic excitatory inputs to rat thalamocortical neurones in vitro. J Physiol (Lond) 510:829-843.

Woolsey TA, Van der Loos H (1970) The description of a cortical field composed of distinct cytoarchitectonic unit. Brain Res 17:205-242.

Wu JY, Guan L, Tsau Y (1999) Propagating activation during oscillations and evoked responses in neocortical slices. J Neurosci 19:5005-5015.

Yuste R, Katz LC (1991) Control of postsynaptic $\mathrm{Ca}^{2+}$ influx in developing neocortex by excitatory and inhibitory neurotransmitters. Neuron 6:333-344.

Zhang ZW, Deschenes M (1997) Intracortical axonal projections of lamina V I cells of the primary somatosensory cortex in the rat: a single-cell labeling study. J Neurosci 17:6365-6379. 